\title{
Photophysics and Charge Generation in Low Energy-offset Blends for Organic Solar Cells
}

\author{
Rishi Shivhare and Natalie Banerji*
}

\begin{abstract}
The power conversion efficiency of organic solar cells has seen a huge improvement in recent years with state-of-the-art solar cells showcasing efficiencies of $\sim 18.5 \%$, which is approaching the performance of inorganic and hybrid-perovskite solar cell technologies. This improvement can be mainly attributed to the discovery of highly efficient donor:acceptor blends with a near-zero energetic offset between the molecular orbital levels of the donor and the acceptor component. A distinctive feature of the high efficiency, low energy-offset blends is that they exhibit a concomitant increase in the short-circuit density and the open-circuit voltage of the solar cell. High open-circuit voltage results from the reduced photon energy loss in the exciton dissociation step, while a high short-circuit current density can be attributed to an efficient charge generation process. The reasons for the efficient exciton dissociation and subsequent separation of Coulomb bound electron-hole pair at negligible driving force is not well understood and, in this short review, we highlight recent results which shed light on the mechanism of charge generation in low energy-offset blends.
\end{abstract}

Keywords: Charge recombination · Charge transfer · Electronic state hybridization · Organic solar cells · Ultrafast spectroscopy

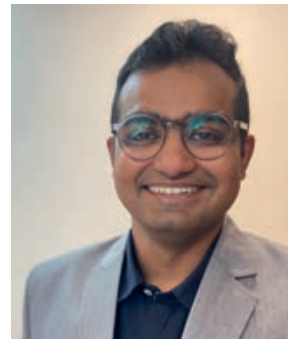

Rishi Shivhare is an FP-RESOMUS postdoctoral fellow in Natalie Banerji's lab at the University of Bern. Previously he completed his $\mathrm{PhD}$ at the Technical University of Dresden, Germany under the supervision of Prof. Stefan C. B. Mannsfeld.

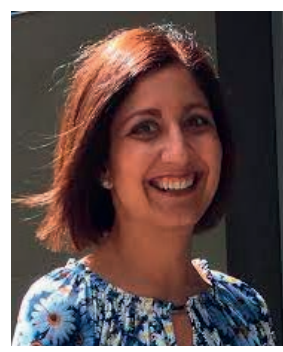

Natalie Banerji is currently a Full Professor of Chemistry at the University of Bern and leads the FemtoMat group. Her research interests include the study of organic and hybrid materials using ultrafast spectroscopic techniques, in view of solar cell and bioelectronic applications. She studied Chemistry at the University of Geneva and obtained her PhD in Physical Chemistry in 2009, under the supervision of Prof. Eric Vauthey. She then moved to the University of California in Santa Barbara (USA), to work on organic solar cells during a post-doctoral stay with Nobel Laureate Prof. Alan J. Heeger (2009-2011)

\section{Introduction}

Organic solar cells (OSCs) are typically composed of a mixture of an electron-donating material (typically a semiconducting polymer) and an electron-accepting compound (a fullerene or non-fullerene based small molecule). The governing idea behind blending two or more materials is to create a heterojunction at the

${ }^{*}$ Correspondence: Prof. Dr. N. Banerji, E-mail: natalie.banerji@unibe.ch Dept. für Chemie, Biochemie und Pharmazie,

University of Bern, Freiestrasse 3, $\mathrm{CH}-3012$ Bern interface between the donor (D) and the acceptor (A) component where photogenerated excitons can dissociate to form free charge carriers. ${ }^{[1,2]}$ Exciton dissociation is a crucial step for generating free charge carriers because in organic semiconductors the exciton binding energy can be as high as $300-500 \mathrm{meV}$, as a result of the low dielectric constant $\left(\varepsilon_{r} \sim 3-4\right)$ of these materials. ${ }^{[3,4]}$ In OSC bulk-heterojunctions, exciton dissociation occurs via a charge transfer reaction at the interface, which leads to the formation of a charge-transfer (CT) state. ${ }^{[5]}$ The interfacial CT state is comprised of an electron-hole pair which resides on different materials (donor and acceptor), but is still coulombically bound. As a next step, the CT state needs to be further dissociated to create free charge carriers which can be collected at the electrodes of the solar cell.

Earlier results suggest that a substantial offset $(\sim 300 \mathrm{meV})$ of the frontier energy levels at the interface (i.e. between the highest occupied molecular orbitals (HOMOs) or lowest unoccupied molecular orbital (LUMOs) of the D/A molecules) is necessary for efficient dissociation of excitons and thus to have a high yield of free charge carriers (Fig. 1a). ${ }^{[6,7]}$ Hence, a majority of first-generation OSCs employed D:A combinations (typically polymer:fullerene blends) with large energy-offsets for efficient charge generation. But this came at a price of losing excess photon energy, defined as: $E_{\text {loss }}=E_{o}-e V_{o c}$, where $E_{g}$ is the optical-gap of the semiconductor and $V_{O C}$ is the open-circuit voltage of the solar cell. A large photon energy loss limits the maximum achievable $V_{O C}$ and hence the overall power conversion efficiency (PCE) of the solar cell (Fig. 1b). Excess photon energy loss was one of the primary reasons why OSCs lagged behind their inorganic and hybrid-perovskite based counterparts. Consequently, PCE values for first-generation OSCs were limited to around $9-10 \%$ in the best case. ${ }^{[8-10]}$

However, in recent years, there have been plenty of reports on D:A blends with efficient charge generation even at near-zero energy level offset ( $\left.E_{\text {offeet }} \sim 50-100 \mathrm{meV}\right)$ of the donor and acceptor materials. ${ }^{[12-14]}$ These findings have led to a new regime in OSCs where efficient charge generation can occur concomitantly at low 
Fig. 1. a) Schematic representation of orbital energy levels for the donor and the acceptor component. Orbital energy offset at the donor:acceptor interface serves as the driving force for the charge transfer process. The energy of the charge-transfer (CT) state roughly scales with the difference between the acceptor LUMO and donor HOMO level. b) Schematic representation of state energy levels and various factors leading to the photon energy loss. Reproduced with permission from ref. [11]. a)

b)

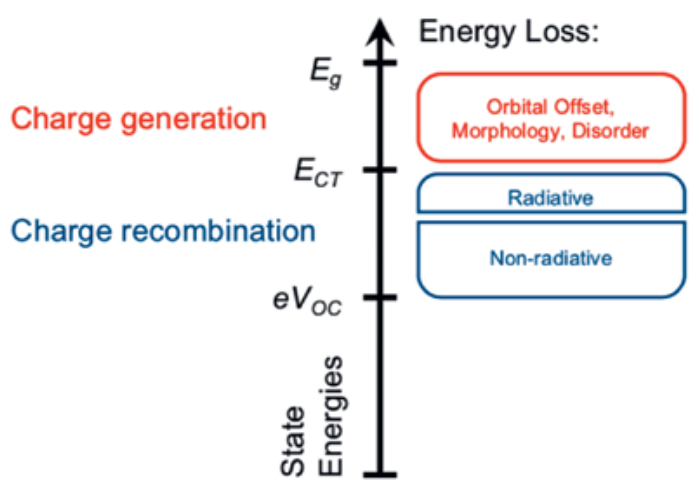

photon energy loss (i.e. high $V_{O C}$ ) and consequently the PCE values for OSCs have steeply increased to around $18-18.5 \%,[15,16]$ which is an improvement of about $100 \%$ compared to firstgeneration OSCs. Most of the high efficiency systems employ non-fullerene acceptors (NFAs), ${ }^{[17]}$ typically composed of fused ring conjugated structures with push-pull effects, most common examples being 3,9-bis(2-methylene-(3-(1,1-dicyanomethylene)indanone) )-5,5,11,11-tetrakis(4-hexylphenyl)-dithieno[2,3$\mathrm{d}: 2$ ', 3'-d']-s-indaceno[1,2-b:5,6-b']dithiophene (ITIC), [18] and 2,2'-((2Z,2'Z)-((12,13-bis(2-ethylhexyl)-3,9-diundecyl12,13-dihydro-[1,2,5] thiadiazolo[3,4-e]thieno[2",3": $\left.4^{\prime}, 5^{\prime}\right]$ thieno[2',3':4,5]pyrrolo[3,2-g]thieno[2',3':4,5] thieno[3,2-b]indole-2,10-diyl)bis(methanylylidene))bis(5,6-difluoro-3-oxo-2,3dihydro-1H-indene-2,1-diylidene))dimalononitrile (BTP-4F) ${ }^{[19]}$ and their derivatives. One of the striking features of the efficient NFA-based systems is that they showcase very high quantum efficiency of charge generation despite near-zero energy offset at the donor:acceptor interface. Also, there have been reports of fullerene-based systems which show similar behavior. The mechanism of charge generation in these systems is not well understood and, in this short review, we aim to highlight the results of recently published works which enable better understanding of the photophysics and charge generation mechanism in low energy-offset OSC systems.

Photon energy loss in OSCs can be divided into two components (Fig. 1b). ${ }^{[11]}$ Firstly, the energy loss associated with charge generation, which can be defined as the difference between the optical gap/singlet $\left(\mathrm{S}_{1}\right)$ energy and the energy of the CT state: $E_{\mathrm{S} 1}-E_{\mathrm{CT}}$. Secondly, the energy loss associated with radiative and non-radiative charge recombination process: $E_{\mathrm{CT}}-\mathrm{e} V_{O C}$. In low energy-offset systems, the $E_{\mathrm{CT}}$ is pushed towards $E_{\mathrm{S} 1}$ in the limit where they become nearly degenerate. As a result, low energyoffset systems minimize the energy loss associated with the charge generation step. Additionally, nearly isoenergetic $S_{1}$ and CT levels can lead to state hybridization and /or mixing of states and this can have interesting implications on the photophysics of such blends. Moreover, higher $E_{\mathrm{CT}}$ values will result in lower nonradiative transition rates as a direct consequence of the energygap law and might also help in reducing energy loss associated with non-radiative charge recombination. We delve into all these implications of employing low energy-offset OSC blends in the following sections.

\section{Low Energy-offset Fullerene-based Solar Cells}

In our recent work, ${ }^{[20]}$ we have investigated a blend of low optical-gap diketopyrropyrrole (DPP) polymer: poly(3- ([2,2':5',2"'-terthiophen]-5-yl)-2,5-bis(6-dodecyloctadecyl)-2,5 dihydropyrrolo[3,4 c]pyrrole1,4-dione-6,5"-diyl), also referred to as $\mathrm{P}(\mathrm{DPP} 6 \mathrm{DOT} 2-\mathrm{T})$, and the fullerene derivative $\mathrm{PC}_{71} \mathrm{BM}$ (Fig. 2a). This system has a very small LUMO-LUMO offset between the DPP polymer and the fullerene molecule and shows a nearzero driving force $\left(E_{\mathrm{S} 1}-E_{\mathrm{CT}} \sim 50 \mathrm{meV}\right)$ for the interfacial electron transfer process. ${ }^{[21]}$ In order to probe charge transfer and charge generation mechanisms in this system, we used a combination of ultrafast spectroscopic techniques and theoretical calculations. Using femtosecond transient absorption (TA) spectroscopy, we probed the excited state lifetime and charge dynamics in this system (Fig. 2b). Firstly, we observed that the $S_{1}$ exciton lifetime in neat P(DPP6DOT2-T) films is very short: of the order of 13-14 ps. This can be attributed to the low optical-gap $\left(E_{\text {opt }} \sim 1.33 \mathrm{eV}\right)$ of the DPP polymer, which results in a high non-radiative decay rate for the exciton due to the energy-gap law. ${ }^{[22]}$ According to the energy-gap law, the non-radiative transition rate increases exponentially with lowering optical-gap of the system. Secondly, the ultrafast emergence (i.e. within $0.1-0.2$ picoseconds) of the charge absorption signature in the TA spectra of P(DPP6DOT2$\mathrm{T}): \mathrm{PC}_{71} \mathrm{BM}$ blend films suggests that electron transfer in this system is ultrafast despite near-zero driving force. We note that charges in CT states and free charges cannot be distinguished by TA spectroscopy.

The occurrence of ultrafast electron transfer is supported by density functional theory (DFT) calculations where the computed rate was found to be in the same range as the experimental value. Additionally, TA data on the blend films revealed significant geminate charge recombination ( $\mathrm{gCR}$ ); gCR refers to the recombination of charges arising from the same CT state. The timescale of gCR ( 13-14 ps) was comparable to the $S_{1}$ exciton lifetime in the neat polymer (Fig. 2c). Since gCR occurs from the CT state, the timescale of gCR is indicative of the CT state lifetime and similar lifetimes for $S_{1}$ and $C T$ states suggest some correlation between the two populations. We identified two key reasons for the correlated $S_{1}$ and $C T$ state lifetimes in this system: i) Due to the close proximity of the energy levels $\left(E_{\mathrm{S} 1} \approx E_{\mathrm{CT}}\right)$, a dynamic equilibrium between the $\mathrm{S}_{1}$ and $\mathrm{CT}$ state populations is established. Depending on the relative magnitudes of forward $\left(S_{1} \rightarrow C T\right)$ and back $\left(\mathrm{CT} \rightarrow \mathrm{S}_{1}\right)$ electron transfer rates, an additional radiative recombination channel is opened up where electrons in the CT state can back transfer to the singlet state and subsequently relax to the ground state $\left(\mathrm{S}_{0}\right)$. This back-electron transfer mechanism explains why the CT state appears to have the same lifetime as the $\mathrm{S}_{1}$ state. ii) Closely lying energy levels of the $S_{1}$ and CT state can lead to electronic-state hybridization. This can in turn increase the oscil- 
a)
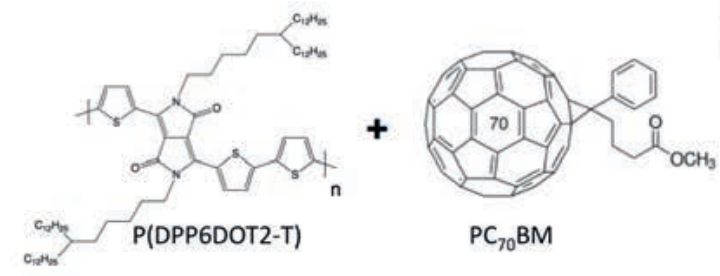

b)

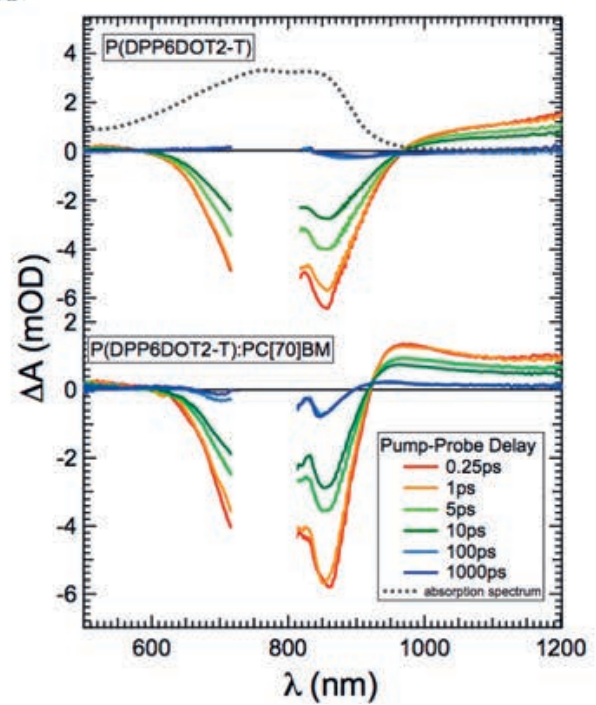

c)

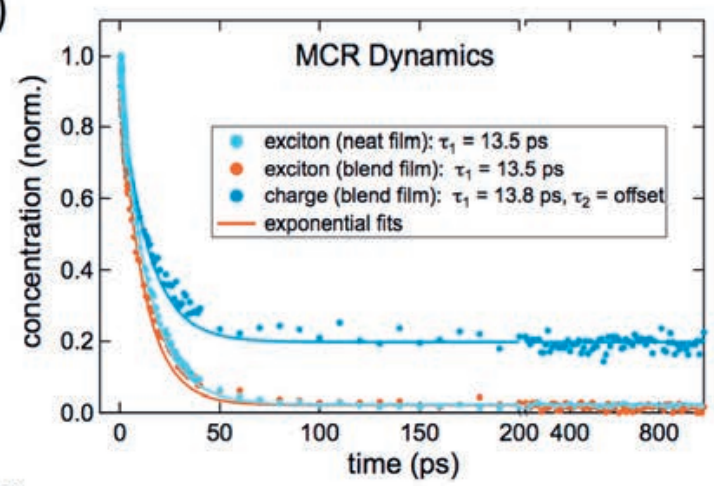

d)

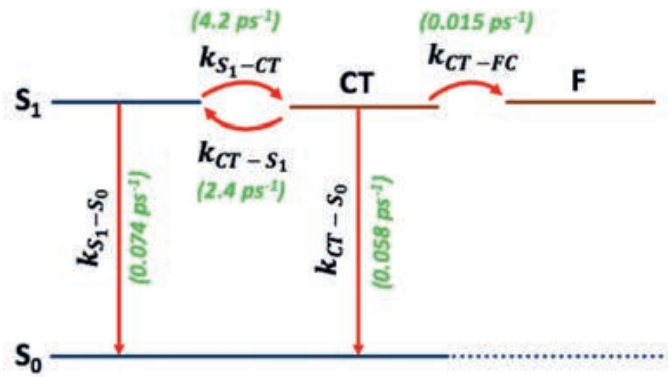

Fig. 2. a) Molecular structure of the DPP polymer: P(DPP6DOT2-T) and the fullerene derivative PC70BM. b) Transient absorption (TA) spectra of the neat DPP polymer (top) and the polymer:fullerene blend film (bottom) at selected pump-probe time delays. c) Decomposed TA dynamics for the exciton and the charge component obtained using multivariate curve resolution (MCR) analysis. d) Jablonski diagram showing various transition rates obtained from kinetic modeling of the TA data. Reproduced from ref. [20].

lator strength for the CT-to-ground state transition as a result of intensity borrowing from the $\mathrm{S}_{1}$ state. Thus, hybridization of $\mathrm{S}_{1}$ and CT states can also explain the correlated lifetimes.

Moreover, kinetic modeling of the TA data revealed relatively slow $(\tau \sim 62 \mathrm{ps})$ dissociation of CT states in the P(DPP6DOT2$\mathrm{T}): \mathrm{PC}_{70} \mathrm{BM}$ blend (Fig. 2d). In traditional polymer:fullerene blends with large energetic-offset, the CT state dissociation is ultrafast. ${ }^{[23]}$ This is mainly attributed to the coupling of charges to delocalized band-like states present in fullerene clusters, which leads to ultrafast separation of charge carriers. ${ }^{[24]}$ However, in the $\mathrm{P}(\mathrm{DPP} 6 \mathrm{DOT} 2-\mathrm{T}): \mathrm{PC}_{70} \mathrm{BM}$ blend, electrons cannot effectively couple to delocalized states, primarily due to the lack of excess energy. In this case, the charges in the CT state have to incoherently hop beyond the Langevin recombination radius $(\sim 5 \mathrm{~nm})$ for the effective dissociation of the CT state. The key parameter governing the incoherent hopping process is the short-range local mobility of charge carriers. In our work, we used electromodulated differential absorption (EDA) spectroscopy, ${ }^{[25]}$ to monitor the short-range mobility of charge carriers. We found that the local mobility in the $\mathrm{P}(\mathrm{DPP} 6 \mathrm{DOT} 2-\mathrm{T}): \mathrm{PC}_{70} \mathrm{BM}$ system is on the lower side which explains the relatively slow dissociation of the CT state. Overall, $\mathrm{S}_{1}$-CT state back-transfer and hybridization resulting in ultrafast gCR combined with slow CT state dissociation were the main discerning features of the $\mathrm{P}(\mathrm{DPP} 6 \mathrm{DOT} 2-\mathrm{T}): \mathrm{PC}_{70} \mathrm{BM}$ system, which directly arise due to the near-zero energetic offset $\left(E_{\mathrm{S} 1}-E_{\mathrm{CT}} \sim 50\right.$ $\mathrm{meV}$ ) between the donor and the acceptor component.

Another polymer:fullerene blend with low energy-offset was reported by Takimiya et al. ${ }^{[12]}$ They investigated a blend of a naphthibisoxadiazole-based polymer (PNOz4T) with the fullerene derivative $\mathrm{PC}_{70} \mathrm{BM}$. Due to the predominantly electron-deficient nature of PNOz4T, it consists of deep lying $\mathrm{HOMO}\left(E_{\text {номо }}=\right.$ $-5.48 \mathrm{eV})$ and LUMO $\left(E_{\text {LUMO }}=-3.65 \mathrm{eV}\right)$ levels and as a result it displays a small LUMO-LUMO offset of $120 \mathrm{meV}$ when blended with $\mathrm{PC}_{70} \mathrm{BM}\left(E_{\mathrm{LUMO}}=-3.77 \mathrm{eV}\right)$. Despite the negligible ener- getic offset for the electron transfer process, the $\mathrm{PNOz} 4 \mathrm{~T}: \mathrm{PC}_{70} \mathrm{BM}$ blend shows impressive device characteristics. PCE values approach $9 \%$ with short circuit current $\left(\mathrm{J}_{\mathrm{SC}}\right) \sim 14.5 \mathrm{~mA} / \mathrm{cm}^{2}$ and $\mathrm{V}_{\mathrm{OC}}$ $\sim 1 \mathrm{~V}$. This system shows an exceptionally low photon energy loss of around $0.52 \mathrm{eV}$ which is at par with values reported for inorganic and perovskite solar cells. As far as the photophysics is concerned, the main findings were similar to those of the above DPP based system. Electron transfer was found to be ultrafast, which was evidenced by the presence of charge absorption signatures at early times in the TA signal. Also, similar to the DPP based blend, the rise of the free charge population happened on a longer timescale of $\sim 100 \mathrm{ps}$. Contrary to the DPP polymers, the singlet lifetime of the PNOz4T polymer was much longer, on the order of 230 ps. Thus, the charge separation process was not limited by fast recombination of carriers and higher efficiency was obtained. The longer singlet lifetime is the primary reason why this system performs better than the DPP:PC ${ }_{70} \mathrm{BM}$ system despite the quite slow charge generation process.

Moreover, the group of Friend et al. reported on the PIPCP:PC ${ }_{60}$ BM blend (Fig. 3a), ${ }^{[26]}$ with low energy offset of molecular orbital levels and near-zero driving force for the interfacial electron transfer process. The group recorded conventional pumpprobe (PP) TA as well as pump-push-probe (PPP) TA spectroscopy data on this system. Using PP-TA spectroscopy, it was found that electron transfer is ultrafast in the PIPCP:PC ${ }_{60} \mathrm{BM}$ blend (Fig. $3 \mathrm{~b}$ ), which is consistent with the findings observed for low energy-offset DPP and PNOz4T based blends. Unlike conventional PP-TA spectroscopy, PPP-TA spectroscopy involves a third pulse (also known as the 'push') which arrives after a certain time delay with respect to the pump pulse and interacts with the photoexcited species in the sample. In the context of the OSC blends, the wavelength of the push pulse is chosen such that it interacts with localized CT states and promotes the CT charges to higher lying electron levels. There, the charges become more delocalized and 
a)

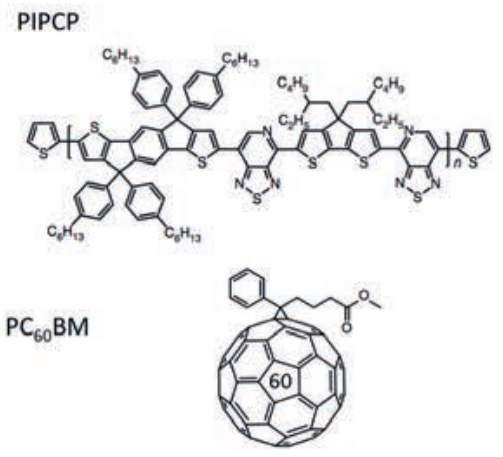

b)

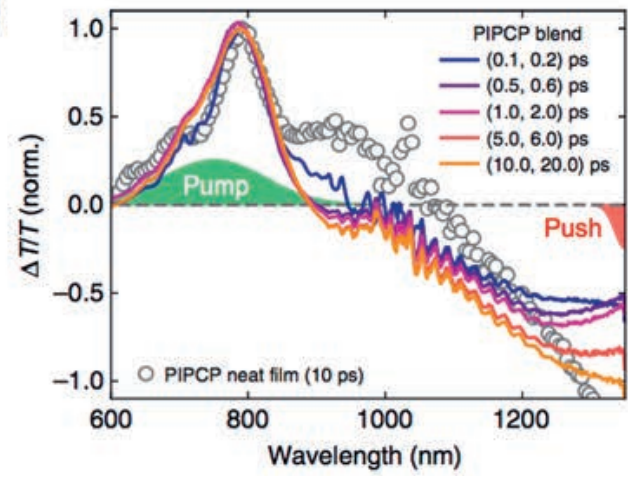

c)

d)
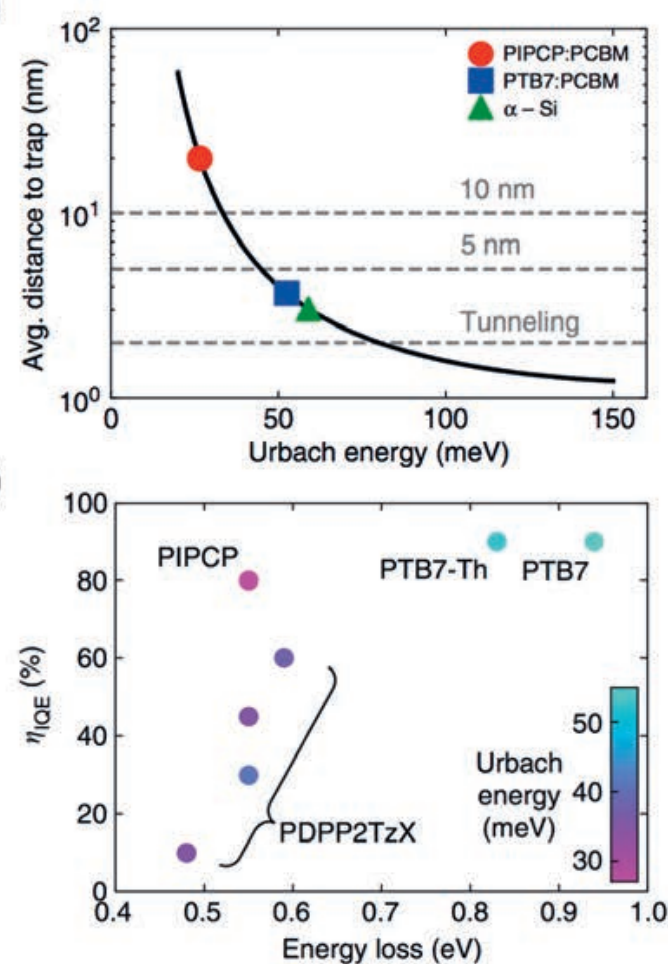

Fig. 3. a) Molecular structure of the donor polymer PIPCP and the fullerene derivative PC ${ }_{60} \mathrm{BM}$. b) TA spectra of the neat PIPCP and the PIPCP:PC ${ }_{60} B M$ blend film at selected time delays. The positive feature centered around $800 \mathrm{~nm}$ corresponds to the ground state bleach (GSB) signal associated with PIPCP excitation while negative features in the near-infrared region correspond to the photoinduced absorption (PIA) signal. c) Computed average distance to CT state trap sites as a function of Urbach energy. In this case, the trap site was defined as a site with energy $>25 \mathrm{meV}$ but $<$ Eg-0.1 eV. d) $\eta_{\mathrm{IQE}}\left(=\mathrm{IQE}_{\mathrm{PV}}\right)$ as a function of photon energy loss $\left(E_{\mathrm{g}}-\mathrm{eV} \mathrm{OC}_{\mathrm{OC}}\right)$ for a variety of polymer:fullerene blends. The color marker denotes the Urbach energy of the system. Reproduced from ref. [26].

are able to easily overcome the coulomb binding energy. As the CT state separates due to the push pulse, the dipolar electric field strength of the corresponding electron-hole pair changes, which affects the electric field on the molecular length scale in the area around the electron-hole pair. These field fluctuations result in changes in the absorption spectrum of the molecules adjacent to the CT state as a result of the Stark effect and lead to the formation of a push-induced electroabsorption (EA) signal.

The push-induced EA signal obtained through PPP spectroscopy provided the timescale for $\mathrm{CT}$ state separation, which was found to be in the range of 1-5 ps for the PIPCP:PC ${ }_{61} \mathrm{BM}$ system. Again, it is apparent that CT state separation is slower in this system compared to high-offset polymer:fullerene blends due to the lack of excess energy, even if it is faster than in the DPP and PNOz4T systems (likely due to higher local mobility). Despite slow CT state separation, the PIPCP system shows an impressive photovoltaic internal quantum efficiency ( $\mathrm{IQE}_{\mathrm{pv}}$ ) of around $80 \%$ (Fig. 3d). $\mathrm{IQE}_{\mathrm{PV}}$ is a measure of yield of free charge carriers extracted per absorbed photon in the solar cell. The authors attribute the high $\mathrm{IQE}_{\mathrm{pV}}$ in the blend to very low interfacial energetic disorder (Urbach energy $<27 \mathrm{meV}$ ) and argue that highly ordered interfaces prevent the CT states from being trapped in lower energy levels in the density of states (DOS, Fig. 3c). As a result, the CT states can effectively separate over longer time periods without undergoing geminate recombination. This study suggests that in general in the low energy offset OSC systems, the Urbach energy should be ideally lower than $30 \mathrm{meV}$ for efficient separation of charges from the CT state.

\section{Low Energy-offset Non-fullerene Acceptor (NFA)-based Solar Cells}

With regards to NFA-based blends, our recent work ${ }^{[27]}$ focused on a low energy-offset system, the J61:m-ITIC blend (Fig. 4a).
This blend has a negligible driving force for the hole transfer $(\mathrm{HT})$ process occurring after photoexcitation of the NFA (HOMOHOMO offset $=0.12 \mathrm{eV}$ ). Using ultrafast TA spectroscopy, we deduced both the electron transfer (ET) and HT rates with different excitation wavelengths. Generally, the time constants obtained from TA spectroscopy are highly multiphasic (given the complex morphology of the donor:acceptor blends) and are not representative of the intrinsic charge transfer rates, due to additional exciton diffusion. In order to overcome this issue, we prepared bilayer planar heterojunction devices and dilute blends $(5: 1 \mathrm{wt} / \mathrm{wt})$, where the acceptor (m-ITIC) was dispersed in the donor (J61) matrix (Fig. 4b). In the dilute blends, the exciton diffusion process is circumvented when the isolated m-ITIC molecules are excited, so that we were able to delineate the intrinsic HT rate from the diffusion-limited process. Irrespective of the device configuration, the fastest HT component was found to be ultrafast ( 0.4-0.9 ps), which was evidenced by a sub-picosecond rise of the charge component in the TA spectra (Fig. 4c,d). The ultrafast HT rate was found to be consistent with the extended Marcus-Levich-Jortner (MLJ) formalism in the limit of moderate electronic coupling and low reorganization energy. We also probed the ET transfer rates by selectively pumping the J61 polymers and we found that the ET rate is even faster than the HT process ( $0.06 \mathrm{ps})$. This was mainly attributed to higher transfer integral $(J)$ values for the ET process than the HT process, as computed for the relaxed dimer geometry using DFT calculations.

Moreover, to study the effect of driving force on the HT at ET rates, we prepared dilute $(5: 1 \mathrm{wt} / \mathrm{wt})$ blends, always using $\mathrm{m}$-ITIC as the acceptor but varying the donor polymer (P3HT, PBTTT, PCDTBT). There was a considerable variation in the energy of the CT state for different blends and hence the driving force for the HT process varied between 0.05 to $0.4 \mathrm{eV}$. Nevertheless, the HT rate was in the sub-picosecond range in all the blends, between 0.5 
a)

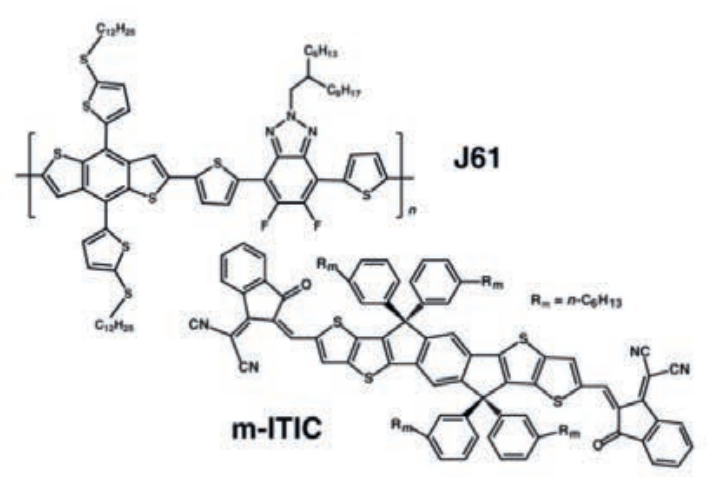

b)

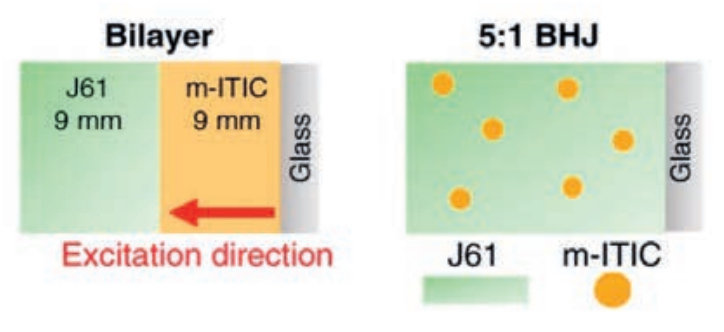

c)

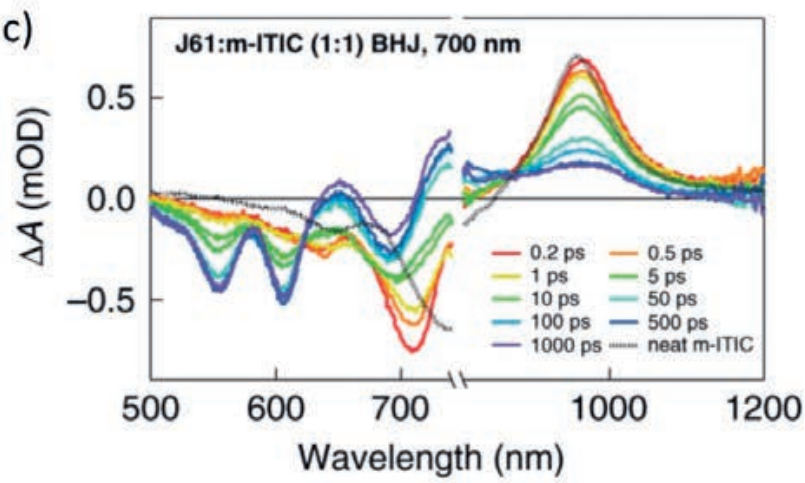

d)

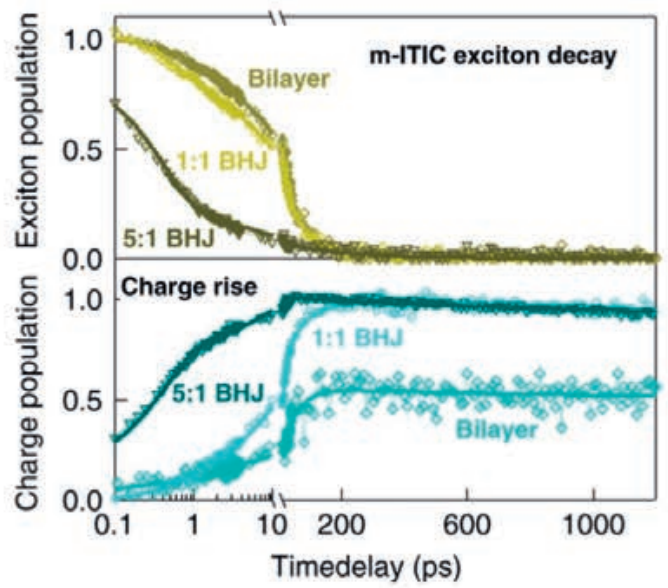

Fig. 4. a) Molecular structure of the polymer J61 and the non-fullerene acceptor (NFA) m-ITIC. b) Schematic representation of the bilayer and the dilute sample configurations. These sample schemes were used to delineate the intrinsic and diffusion-mediated charge transfer rate. $\mathrm{c}$ ) TA spectra of the J61:m-ITIC blend film (1:1 weight ratio, excitation of m-ITIC) at selected pump-probe delay times. d) TA dynamics showing decay of the m-ITIC exciton population and the concomitant rise of the charge population at ultrafast time scale $(<0.9 p s)$. The fastest charge rise component was found to be ultrafast in all configurations (bilayer, dilute and normal 1:1 wt/wt blend) suggesting the intrinsic hole transfer rate in this system is ultrafast. Reproduced from ref. [27].

to $0.08 \mathrm{ps}$. Again, the ET process was found to be faster (generally $<0.1 \mathrm{ps}$ ) than the HT process. Overall, our work suggests that the driving force in itself is not a limiting factor for the ET/HT process, since the intrinsic ET/HT rate is in the sub-picosecond range irrespective of the energetic offset. Thus, the low energy-offset blends are a promising avenue for furthering the performance of OSCs given that the interfacial molecular orientation of the donor/ acceptor and the overall bulk-heterojunction (BHJ) morphology can be optimized.

\section{Discussion and Conclusions}

As evidenced by our recent work and other reports, low energy-offset blends are promising for next-generation OSCs, where possibly the benchmark for $20 \%$ PCE will be breached in the coming years. Ultrafast spectroscopic measurements provide ample evidence that the intrinsic charge transfer process in such blends remains ultrafast despite the small driving force. However, the subsequent separation of Coulomb-bound electron-hole pair (in the CT state) slows down and occurs in tens of picoseconds. Such a scenario demands that the donor and acceptor materials possess long $\mathrm{S}_{1}$ and CT state lifetimes in order to ensure that CT state separation outcompetes charge recombination. Thus, from the synthetic chemists' perspective, the focus should be on developing novel donor and acceptor materials with long excited state lifetimes and reduced non-radiative recombination from the CT state, which will ensure efficient generation of free charge carriers despite relatively slow separation of charges in the CT state. On the other hand, our future work will focus on further understanding the photophysics and charge generation mechanism in such systems using a combination of ultrafast spectroscopic measurements and theoretical calculations.

Received: August 31, 2021

[1] G. Yu, J. Gao, J. C. Hummelen, F. Wudl, A. J. Heeger, Science 1995, 270, 1789, https://doi.org/10.1126/science.270.5243.1789

[2] G. Li, R. Zhu, Y. Yang, Nature Photon. 2012, 6, 153, https://doi.org/10.1038/nphoton.2012.11

[3] M. Knupfer, Appl. Phys. A 2003, 77, 623, https://doi.org/10.1007/s00339-003-2182-9

[4] M. Engel, F. Kunze, D. C. Lupascu, N. Benson, R. Schmechel, Physica Status Solidi (RRL)-Rapid Research Letters 2012, 6, 68, https://doi.org/10.1002/pssr.201105488

[5] C. Deibel, T. Strobel, V. Dyakonov, Adv. Mater. 2010, 22, 4097, https://doi.org/10.1002/adma.201000376

[6] S. Günes, H. Neugebauer, N. S. Sariciftci, Chem. Rev. 2007, 107, 1324, https://doi.org/10.1021/cr050149z

[7] A. A. Bakulin, A. Rao, V. G. Pavelyev, P. H. van Loosdrecht, M. S. Pshenichnikov, D. Niedzialek, J. Cornil, D. Beljonne, R. H. Friend, Science 2012, 335, 1340, https://doi.org/10.1126/science.1217745

[8] C. H. Peters, I. Sachs-Quintana, J. P. Kastrop, S. Beaupre, M. Leclerc, M. D. McGehee, Adv. Ener. Mater. 2011, 1, 491, https://doi.org/10.1002/aenm.201100138

[9] W. Huang, E. Gann, L. Thomsen, C. Dong, Y. B. Cheng, C. R. McNeill, Adv. Ener. Mater. 2015, 5, 1401259, https://doi.org/10.1002/aenm.201401259

[10] Y. Zheng, G. Wang, D. Huang, J. Kong, T. Goh, W. Huang, J. Yu, A. D. Taylor, Solar RRL 2018, 2, 1700144, https://doi.org/10.1002/solr.201700144

[11] S. M. Menke, N. A. Ran, G. C. Bazan, R. H. Friend, Joule 2018, 2, 25, https://doi.org/10.1016/j.joule.2017.09.020

[12] K. Kawashima, Y. Tamai, H. Ohkita, I. Osaka, K. Takimiya, Nat. Commun. 2015, 6, 1, https://doi.org/10.1038/ncomms10085 
[13] J. Liu, S. Chen, D. Qian, B. Gautam, G. Yang, J. Zhao, J. Bergqvist, F. Zhang, W. Ma, H. Ade, Nat. Ener. 2016, 1, 1, https://doi.org/10.1038/nenergy.2016.89

[14] N. A. Ran, J. A. Love, C. J. Takacs, A. Sadhanala, J. K. Beavers, S. D. Collins, Y. Huang, M. Wang, R. H. Friend, G. C. Bazan, Adv. Mater. 2016, 28, 1482, https://doi.org/10.1002/adma.201504417

[15] Q. Liu, Y. Jiang, K. Jin, J. Qin, J. Xu, W. Li, J. Xiong, J. Liu, Z. Xiao, K. Sun, Science Bull. 2020, 65, 272, https://doi.org/10.1016/j.scib.2020.01.001

[16] Z. Chen, W. Song, K. Yu, J. Ge, J. Zhang, L. Xie, R. Peng, Z. Ge, Joule 2021 https://doi.org/10.1016/j.joule.2021.06.017

[17] J. Hou, O. Inganäs, R. H. Friend, F. Gao, Nat. Mater. 2018, 17, 119 https://doi.org/10.1038/nmat5063

[18] Y. Lin, J. Wang, Z. G. Zhang, H. Bai, Y. Li, D. Zhu, X. Zhan, Adv. Mater 2015, 27, 1170, https://doi.org/10.1002/adma.201404317

[19] J. Yuan, Y. Zhang, L. Zhou, G. Zhang, H.-L. Yip, T.-K. Lau, X. Lu, C. Zhu, H. Peng, P. A. Johnson, M. Leclerc, Y. Cao, J. Ulanski, Y. Li, Y. Zou, Joule 2019, 3, 1140, https://doi.org/10.1016/j.joule.2019.01.004

[20] R. Shivhare, G. J. Moore, A. Hofacker, S. Hutsch, Y. Zhong, M. Hambsch, T. Erdmann, A. Kiriy, S. C. Mannsfeld, F. Ortmann, N. Banerji, Adv. Mater. 2021, 2101784, https://doi.org/10.1002/adma.202101784

[21] R. Shivhare, T. Erdmann, U. Hörmann, E. Collado-Fregoso, S. Zeiske, J. Benduhn, S. Ullbrich, R. Hübner, M. Hambsch, A. Kiriy, B. Voit, D. Neher, K. Vandewal, S. C. B. Mannsfeld, Chem. Mater. 2018, 30, 6801, https://doi.org/10.1021/acs.chemmater.8b02739

[22] R. Englman, J. Jortner, Mol. Phys. 1970, 18, 145 https://doi.org/10.1080/00268977000100171

[23] A. C. Jakowetz, M. L. Böhm, J. Zhang, A. Sadhanala, S. Huettner, A A. Bakulin, A. Rao, R. H. Friend, J. Am. Chem. Soc. 2016, 138, 11672 https://doi.org/10.1021/jacs.6b05131
[24] S. Gélinas, A. Rao, A. Kumar, S. L. Smith, A. W. Chin, J. Clark, T. S. Van Der Poll, G. C. Bazan, R. H. Friend, Science 2014, 343, 512, https://doi.org/10.1126/science.1246249

[25] J. De Jonghe-Risse, M. Scarongella, J. C. Brauer, E. Buchaca-Domingo, J.-E. Moser, N. Stingelin, N. Banerji, Nat. Commun. 2016, 7, 1, https://doi.org/10.1038/ncomms12556

[26] S. M. Menke, A. Cheminal, P. Conaghan, N. A. Ran, N. C. Greehnam, G. C. Bazan, T.-Q. Nguyen, A. Rao, R. H. Friend, Nat. Commun. 2018, 9, 1 , https://doi.org/10.1038/s41467-017-02457-5

[27] Y. Zhong, G. J. Moore, P. Krauspe, B. Xiao, F. Günther, J. Kublitski, R. Shivhare, J. Benduhn, E. BarOr, S. Mukherjee, K. M. Yallum, J. Réhault, S. C. B. Mannnsfeld, D. Neher, L. J. Richter, D. M. DeLongchamp, F. Ortmann, K. Vandewal, E. Zhou, N. Banerji, Nat. Commun. 2020, 11, 1, https://doi.org/10.1038/s41467-020-14549-w

\section{License and Terms}

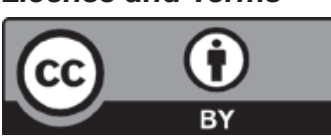

This is an Open Access article under the terms of the Creative Commons Attribution License CC BY 4.0. The material may not be used for commercial purposes.

The license is subject to the CHIMIA terms and conditions: (http:// chimia.ch/component/sppagebuilder/?view=page \&id=12).

The definitive version of this article is the electronic one that can be found at https://doi.org/10.2533/chimia.2021. 